\title{
Physical and chemical characterization of cerium(IV) oxide nanoparticles
}

\author{
Gemma Cepriá $^{1}$ - Walvin R. Córdova ${ }^{1}$ - Oscar Céspedes ${ }^{2} \cdot$ Laura Sánchez-García $^{1}$. \\ Pilar Ferrer $^{3}$ • Diego Gianolio ${ }^{3}$. Juan R. Castillo ${ }^{1}$
}

\begin{abstract}
Chemical composition, size and structure of the nanoparticle are required to describe nanoceria. Nanoparticles of similar size and Ce(III) content might exhibit different chemical behaviour due to their differences in structure. A simple and direct procedure based on affordable techniques for all the laboratories is presented in this paper. The combination of Raman and UV-vis spectroscopy and particle impact coulometry (PIC) allows the characterization of nanoceria of small size from 4 to $65 \mathrm{~nm}$ at a concentration from micromolar to nanomolar, a concentration range suitable for the analysis of lab-prepared or commercial nanoparticle suspensions, but too high for most analytical purposes aimed at nanoparticle monitoring. While the PIC limits of size detection are too high to observe small nanoparticles unless catalytic amplification is used, the method provides a simple means to study aggregation of nanoparticles in the media they are needed to be dispersed for each application. Raman spectroscopy provided information about structure of the nanoparticle, and UV-vis about their chemical behaviour against some common reducing and oxidizing agents.
\end{abstract}

Keywords Nanoceria $\cdot$ Chemical behaviour · Raman · Particle impact coulometry $\cdot$ XAS

Gemma Cepriá

gcepria@unizar.es

1 Analytical Spectroscopy and Sensors Group (GEAS), Environmental Sciences Institute (IUCA), Analytical Chemistry Department, University of Zaragoza, C/Cerbuna 12, 50009 Zaragoza, Spain

2 School of Physics and Astronomy, University of Leeds, LS2 9JT Leeds, UK

3 Diamond Light Source, Harwell Science and Innovation Campus, Chilton, Didcot OX11 0DE, UK

\section{Introduction}

Cerium(IV) oxide nanoparticles or nanoceria are used in a broad range of disciplines from biomedical (e.g. ophthalmology) to technological applications (e.g. catalyst in redox processes), and in the preservation of surfaces against oxidation or UV irradiation [1]. As their use and significance grow, nanoceria leaking becomes a key issue with serious repercussions both for human health and the environment. However, to assess the risks posed by nanoceria, different factors are relevant. These include not only their concentration but also properties such as the nanoparticles' shape, size and structure, as they affect their reactivity and might affect their toxicity [2]. The relevance of the nanoparticle size and surface charge and composition has been noticed by many different authors previously. Nanoparticles with a size below $25 \mathrm{~nm}$ can cross the blood-brain barrier, whereas bigger particles will typically accumulate in the liver and/or be discarded through the kidneys. Therefore, only particles smaller than this size can become toxic to the central neural system where the barrier plays a critical role [3]. From the point of view of drug therapy and magnetic resonance, these smaller particles can be used for the targeted treatment and magnetic imaging of brain diseases with the appropriate functionalization and making use of their larger magnetic susceptibility $[4,5]$.

The surface and oxidation state will also be critical to determine their potential to cause biological damage directly [6] or through oxidative stress which in turn can lead to cancer and other diseases via free radicals [7, 8]. Recently, it has been demonstrated that the $\mathrm{Ce}$ (III) content present on the surface of the nanoparticle is a decisivet factor to asses the biotoxicity of nanoceria [9].

Shape and size have been usually characterized using transmission electron microscopy (TEM), whereas X-ray diffraction (XRD) provides information about chemical composition 
and crystal structure. Raman spectroscopy has been suggested as well as a tool to derive the size of the nanoparticles, since the $460 \mathrm{~cm}^{-1}$ band-half width at half height (HWHM) is related to their diameter [10]. However, a critical drawback of these techniques is that to perform such tests the nanoparticles need to be extracted from their suspended media, making difficult their study in solution. Dynamic light scattering (DLS) and nanoparticle tracking analysis (NTA) are performed in suspension and can also provide size information, but in this case, the diameter obtained is dependent on the media in which it has been measured. Furthermore, DLS cannot be used for small nanoparticles or transparent media [11], rendering null its applications to estimate ceria leakage in natural water sources.

For the study of nanoceria redox properties, UV-vis spectroscopy can be a very affordable and simple tool as the UVvis spectra change with the $\mathrm{Ce}(\mathrm{III}) / \mathrm{Ce}(\mathrm{IV})$ ratio on the nanoparticle surface [12]. Some redox reactions with colourants and dyes have been followed this way [13], but the required concentration of nanoparticles is $2.5 \mu \mathrm{g} / \mathrm{mL}\left(510^{15} \mathrm{NP} / \mathrm{L}\right.$, $8.3 \mathrm{nM}$ ), that makes it only useful for characterizing concentrated samples, such as standard solutions or lab-prepared nanoparticles.

Electroanalytical techniques like particle impact coulometry (PIC) $[14,15]$ allow the detection and monitoring of nanoparticles in suspensions, showing the way to study and understand the behaviour and interactions of nanoparticles in the media in which they are suspended [16]. Recent studies and developments of this technique have improved their analytical performance lowering the limits of detection $[17,18]$ down to $10^{8}$ nanoparticles/L $(0.2 \mathrm{fM})$ and offering a reliable way to calculate concentration and size of nanoparticles in suspension, even in opaque solutions that contain other nanoparticles [19]. These points marked this technique as a unique and promising mean to obtain valuable information of nanoparticles in complex samples without extraction, separation or use of chemical treatment. However, the presence of absorbed species (molecules or other nanoparticles) on the surface of the PIC working electrode poses a serious problem that hinders the correct development of its applications [20]. On the other hand, it is possible to attain lower limits of detection by diminishing the noise improving the electronic design of the instrumentation and using carbon fibre microelectrodes [21], although these improvements have not been applied yet to non-synthetic samples, but only to nanoparticles-spiked seawater [16].

In this work, we address the physico-chemical characterization of nanoceria samples (two aqueous suspensions commercialized for chemical applications and one sample for polishing purposes) by Raman and UV-vis spectroscopy, TEM, X-ray absorption near edge structure (XANES) and PIC. Key properties such as shape, size, redox and chemical structure are studied with the aim of understanding the chemical reactivity of the nanoparticles. We propose a simple methodological alternative based on Raman, UV-vis and PIC to characterize nanoceria and to obtain the maximum information with the minimum time investment to be affordable for most laboratories and users. We think this would be very useful for researchers that need to select a cerium oxide nanoparticle for catalytic or biomedical experiments and also will help to predict the reactivity that may harm living organism.

\section{Experimental}

\section{Samples}

Three nanoceria aqueous suspensions of different nominal diameter were studied: a $4 \mathrm{~nm}$ standard (STD-4) from PlasmaChem Gmbh $\left(5 \% w / w\right.$ in $\mathrm{HNO}_{3}$, density of $1.04 \mathrm{~g} /$ $\mathrm{mL}$ ), a 10-20 nm standard (STD-10) from Sigma Aldrich $(20 \% w / w$ in $2.5 \% w / w$ acetic acid, density of $1.22 \mathrm{~g} / \mathrm{mL}$ ) and a $30 \mathrm{~nm}$ suspension (STD-30) form Alfa Aesar (25\% w/w in $\mathrm{H}_{2} \mathrm{O}$ at $\mathrm{pH} 3$, density unspecified) used for polishing purposes. The colloidal suspensions were kept in the dark at $4{ }^{\circ} \mathrm{C}$ and sonicated for $5 \mathrm{~min}$ before use. Dilutions were prepared by suspending aliquots of the bulk suspensions in the same electrolyte in which they were delivered. All dilutions were prepared daily and sonicated for $2 \mathrm{~min}$ before use.

All reagents were of analytical grade. All solutions were prepared with ultrapure water (Milli Q Advantage).

UV-vis spectra of the nanoparticles were periodically obtained in $0.1 \mathrm{M} \mathrm{HCl}$ to detect chemical changes or degeneration of the standard.

\section{Instruments and methods}

TEM was used to characterize the core size and shape of the nanoparticles. Cerium oxide nanoparticles were imaged using a TEM (Tecnai G2-F30 Field Emission Gun microscope) working at $300 \mathrm{kV}$ with a super-twin lenses and $0.2 \mathrm{~nm}$ point-to-point resolution and 0.1 line resolution. The microscope is equipped with an energy-dispersive X-ray analysis system (INCA 200 X-Sight, Oxford Instruments) for elemental analysis and element localization on samples. All samples were prepared on a carbon-coated 300 mesh copper grid. The liquid samples were prepared by depositing and air-drying a droplet on the TEM grid. Images were recorded using a GATAN CCD camera and subsequently analysed with ImageJ 1.47 V. (National Institute of Health, USA) to obtain the size of individual nanoparticles. For each standard, five images were analysed and at least 100 particles/image examined (i.e. 500 nanoparticles/sample analysed in total) for calculating the nanoceria size histograms using Excel. The shape heterogeneity of the ceria nanoparticles in STD-30 precluded us to obtain a representative histogram of the size population 
in this sample, thus reporting the size as a range here (see Table 1).

X-ray absorption spectroscopy (XAS) provides geometrical and electronic information of different materials. XAS spectra are divided in two regions: the XANES and the extended X-ray absorption fine structure (EXAFS). Here, XAS was employed to gain insight into the oxidation state of cerium in the standard ceria nanoparticles, being thus focused on the Ce-Le3 edge region $(E=5526-6156 \mathrm{eV}$ with $0.1 \mathrm{eV}$ interval). The XAS measurements were performed at room temperature on the B18 beamline at Diamond Light Source (UK) [22]. Data were collected in $\mathrm{CeO}_{2}$ NPs in the fluorescence yield mode with a nine-element Germanium detector using a double crystal Si111 monochromator and Pt-coated branch. Harmonics were rejected with a pair of harmonic rejection mirrors. The Ce-L $\alpha 1$ fluorescence line was recorded with the detector placed parallel to the X-ray electric polarization field (i.e. forming $90^{\circ}$ from the incoming X-ray beam). Data treatment was achieved with ATHENA software [23].

Raman spectroscopy addressed the characterization of the size and structure of the nanoparticles. Here, the ceria nanoparticles were precipitated by increasing the ionic strength of the solution with $\mathrm{NaCl}$ and centrifuged at $5000 \mathrm{rpm}$ for $10 \mathrm{~min}$. The aqueous supernatant was discarded and the solid was shaken with water and centrifuged twice at $5000 \mathrm{rpm}$ for $10 \mathrm{~min}$ for cleaning purposes. The precipitated nanoparticles were allowed to dry at $60^{\circ} \mathrm{C}$ and Raman spectra were obtained with a LabRAM HR 800, Horiba Scientific, with a blue laser $(\lambda=473 \mathrm{~nm})$ and $250 \mathrm{~mW}$ maximum power, spot size $0.5-1 \mu \mathrm{m}$.

UV-vis spectroscopy was applied to obtain the redox state of ceria in the nanoparticles suspensions, where differences in the UV-vis spectra may be related to different ratios of $\mathrm{Ce}$ (III) and $\mathrm{Ce}(\mathrm{IV})$ in the samples. UV-vis spectra of the colloidal dispersions were obtained in different media $(\mathrm{HCl}$, hydrogen peroxide and ascorbic acid) with an Agilent 8453 spectrophotometer between 200 and $1100 \mathrm{~nm}$.

PIC was employed to study the nanoparticles reactivity and stability in suspension. The electrochemical measurements were carried out with a computer controlled AutoLab

Table 1 Nanoparticle radii derived by different techniques

\begin{tabular}{lllll}
\hline sample & \multicolumn{2}{l}{ Size-diameter (nm) } & & $\begin{array}{l}\text { Ce(III) content } \\
\text { XANES }\end{array}$ \\
\cline { 2 - 4 } & Raman & TEM & PIC & XAN \\
\hline STD-4 & n.m. & 3 & $8^{\mathrm{a}}$ & $9 \%$ \\
STD-10 & n.m. & 10 & $<54^{\mathrm{b}}$ & $10 \%$ \\
STD-30 & 14 to 64 & 2 to 65 & $<50^{\mathrm{b}}$ & $6 \%$ \\
\hline
\end{tabular}

n.m. not measured

${ }^{\text {a }}$ PIC data obtained using catalytic amplification

${ }^{\mathrm{b}}$ Data obtained by direct reduction of the nanoparticle on the electrode
PGSTAT-12 potentiostat (Utrecht, The Netherlands) connected to a $20-\mathrm{mL}$ voltammetric cell with an $\mathrm{Ag} / \mathrm{AgCl} / 3 \mathrm{M} \mathrm{NaCl}$ reference electrode from $\mathrm{BASi}$, a $\mathrm{Pt}$ auxiliary electrode and working microelectrodes of carbon fibre and $\mathrm{Pt}(11 \pm 2 \mu \mathrm{m}$ and $10 \mu \mathrm{m}$ diameter, respectively) (BASi). The electrochemical cell was kept in a Faraday cage during the analyses.

Before measurement, the carbon fibre microelectrode was polished with alumina and the platinum microelectrode with two diamond powders ( 3 and $6 \mu \mathrm{m}$ ), then they were rinsed with deionized water and sonicated for $5 \mathrm{~min}$ in deionized water. While the carbon fibre microelectrode was ready to use, the Pt microelectrode still required a chemical treatment with a solution of $1 \mathrm{mM} \mathrm{NaBH}_{4}$ in $1 \mathrm{M} \mathrm{HCl}$ for $15 \mathrm{~min}$ to eliminate the platinum oxides from the surface and minimize the noise.

The data were treated using a Fast Fourier transform filter that blocked the $50-$ and $150-\mathrm{Hz}$ frequencies, as well as to count the transients and calculate their peak area.

\section{Results and discussion}

\section{Physical characterization}

TEM and Raman spectroscopy provided information about shape, size and structure of the nanoparticle once they have been isolated from the solution.

The crystalline structure and spherical shape of the $\mathrm{CeO}_{2}$ nanoparticles could be clearly appreciated from the TEM bright-field images of STD-4 and STD-10. The TEM-based size histograms showed diameter distributions in the commercial suspensions ranging from 1 to $5 \mathrm{~nm}$ (most frequent size $3 \mathrm{~nm}$ ), and from 2 to $11 \mathrm{~nm}$ (most frequent size $5 \mathrm{~nm}$ ) for STD-4 and STD-10, respectively (Fig. 1a, b). STD-30 was observed to be a suspension of cubic-shaped nanoparticles and great dispersion of size (Fig. 1c), where their diameters range from 2 to $65 \mathrm{~nm}$. The particle-core sizes determined by TEM differed considerably from the nominal size reported by the manufacturer for STD-10 (i.e. 5 versus 10-20 nm), and showed a wider range of variation relative to the nominal size for STD-30 (i.e. 2-65 versus $30 \mathrm{~nm}$ ). The discrepancy was not that notorious for STD-4 (3 versus $4 \mathrm{~nm}$ ).

Raman spectroscopy was used to investigate the chemical configuration of the samples and nanoparticles dimensions. All the three standard suspensions showed the characteristic F2g peak at $465 \mathrm{~cm}^{-1}$ (Fig. 2) that corresponds to a symmetrical stretching mode of the $\mathrm{Ce}-\mathrm{O}$ bond. The width at half height of this peak can be used to calculate the diameter of the nanoparticle (HWHM) [10] and the oxygen vacancies concentration that defines the chemical properties of nanoceria [24]. Significant differences in this band could be observed amongst the three nanoparticles. The band exhibited a lorentzian profile for STD-30, but a more complex structure 

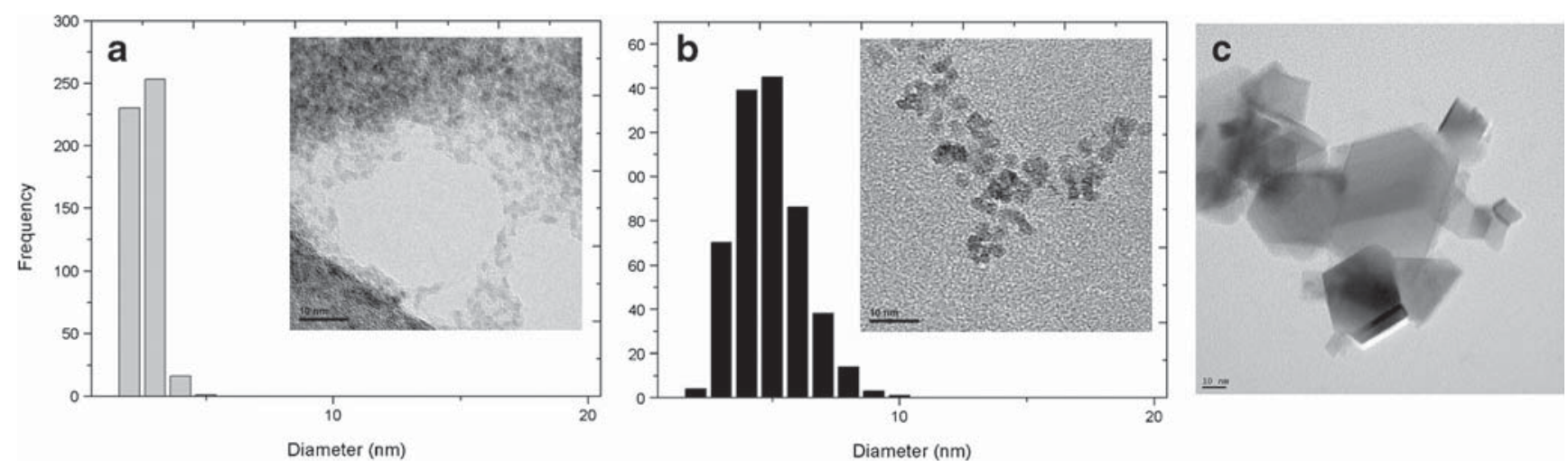

Fig. 1 Histograms of the nanoparticles size distributions in STD-4 (a) and STD-10 (b), derived from the analysis of TEM images (inserts). TEM image of STD-30 (c)

for STD-4 and STD-10 that consisted of an asymmetric shift and a widening of the F2g peak. This widening is attributed to a change in the lattice constant due to vacancies and oxygen content $[25,26]$. The shape of the Raman spectra in the STD-4
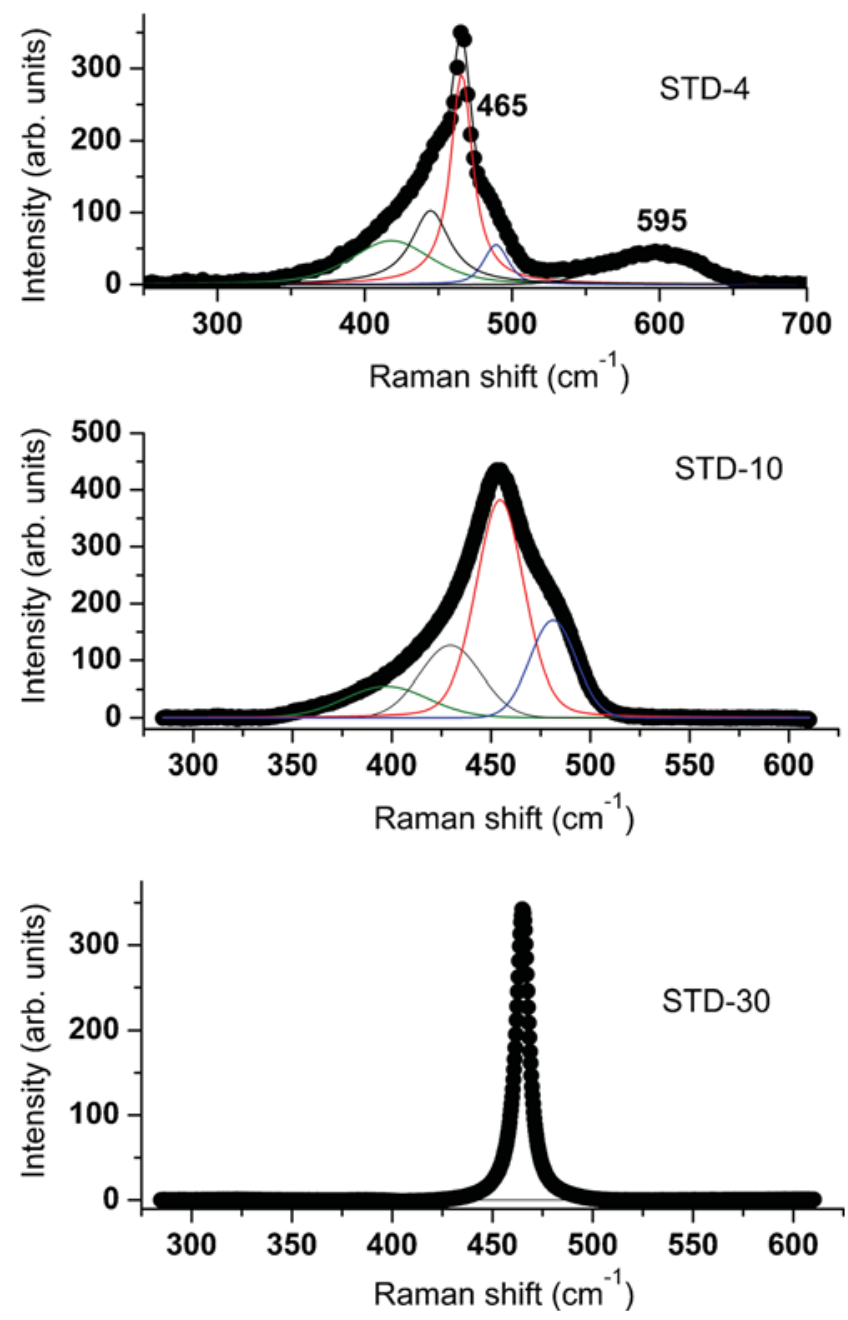

Fig. 2 Raman spectra of STD-4, STD-10 and STD-30 showing the complex structure of the two first samples and STD-10 F2g zone (Fig. 2) follows the behaviour observed in small nanoparticles, with the STD-4 spectrum being similar to that of porous nanorods used in oxygen storage [27]. The Raman spectrum is very sensitive to local heating due to the laser used to measure the spectra, itself with a size of $1 \mu \mathrm{m}$. The thermal conductivity will vary depending on micro-scale changes in aggregation or thickness of the sample, leading to the variations in Raman spectra as a function of the focal point in the Raman microscope. Consequently, different parts of the STD-4 and STD-10 samples appear to have very different Raman spectra, but they can be fitted using the same peak analysis. We can consider that the F2g peak remains roughly in the same position, but its width can drastically change from $\sim 10$ to $35 \mathrm{~cm}^{-1}$ as a function of the inhomogeneous, local strain [26].

In addition to $465 \mathrm{~cm}^{-1}$ for STD- 4 and $455 \mathrm{~cm}^{-1}$ for STD10 , other peaks appeared at $\sim 380,420$ and $490 \mathrm{~cm}^{-1}$ in STD-4 (Fig. 2a) and 396, 429 and $481 \mathrm{~cm}^{-1}$ in the STD-10 Raman spectra (Fig. 2). For the three measurements applied on STD-4 and STD-10, the F2g peak overlapped depending on its peak width, resulting in a shift of the spectra maximum from 465 to $450-455 \mathrm{~cm}^{-1}$. The peaks at $\sim 380,420$ and $490 \mathrm{~cm}^{-1}$ in the STD-4 and 396, 429 and $481 \mathrm{~cm}^{-1}$ in STD-10 can be attributed to the transversal and longitudinal phonons at the L symmetry point of the Brillouin zone [27, 28]. Another peak at $595 \mathrm{~cm}^{-1}$ was also observed in STD-4. This signal is sometimes referred to as the "defect peak", commonly assigned to intrinsic defects and oxygen vacancies [29, 30]. The areal ratio between the $\mathrm{F} 2 \mathrm{~g}$ peak at $465 \mathrm{~cm}^{-1}$ and this defect peak can therefore be used to estimate the crystallinity and oxygen content of the sample. Here, the ratio was approximately $1: 1$ if only the deconvoluted band at $450 \mathrm{~cm}^{-1}$ is considered, while six to seven times larger when calculated relative to the whole area integrated under the peaks at 380, 420, 450, 465 and $490 \mathrm{~cm}^{-1}$. The enhanced factor when considering the whole area is likely caused by a broadening of the bands due to inhomogeneity between the particles and the heating effects aforementioned, therefore the F2 $\mathrm{g}$ to defect peak ratio is more 
likely to be representative of the actual crystallinity and oxygen content of the sample.

The Raman spectra for sample STD-30 offered a clear and narrow band at $465 \mathrm{~cm}^{-1}$ (Fig. 2), whose HWHM depended on the area at which aimed the laser beam varying from 8.5 to $5.8 \mathrm{~cm}^{-1}$. A broad distribution of size, from 14 to $64 \mathrm{~nm}$, was observed as it was also confirmed by TEM. The size calculation was not carried out for STD-4 and STD-10 due to the complex structure of the band.

\section{Chemical characterization}

XAS provided information about the chemical composition of the samples and UV-vis and PIC were used to obtain information of the nanoparticles in suspension.

Analysis of the XAS region provided information on the cerium oxidation state in the nanoceria suspensions. The presence of two peaks in the excitation-energy signal at the Ce L3 edge suggested the predominance of $\mathrm{Ce}(\mathrm{IV})$ in the three samples (Fig. 3).

Applying a linear combination fitting (LCF) to the energy signal provides an estimate for the proportion of the Ce oxidation state and confirms the predominance of Ce(IV). Two cerium species were used as references of the two oxidation states of cerium, $\mathrm{CeO}_{2}$ and $\mathrm{Ce}\left(\mathrm{NO}_{3}\right)_{3}$, respectively. The $\mathrm{LCF}$ results for Ce L3-edge XANES spectra indicated that $\mathrm{Ce}$ (IV) was the dominant form of Ce ( $\geq 90 \%)$ in the three suspensions, with the contribution of $\mathrm{Ce}$ (III) varying in function of the sample (Table 1). The lower proportion of less-packed $\mathrm{Ce}(\mathrm{III})$ observed in STD-30 (6 \%) relative to STD-4 (9\%) or STD-10 (10\%) was interpreted in relation to the larger size of the ceria nanoparticles in STD-30 (Table 1). This confirms the lower content of $\mathrm{Ce}(\mathrm{III})$ in the larger STD-30 nanoparticles.

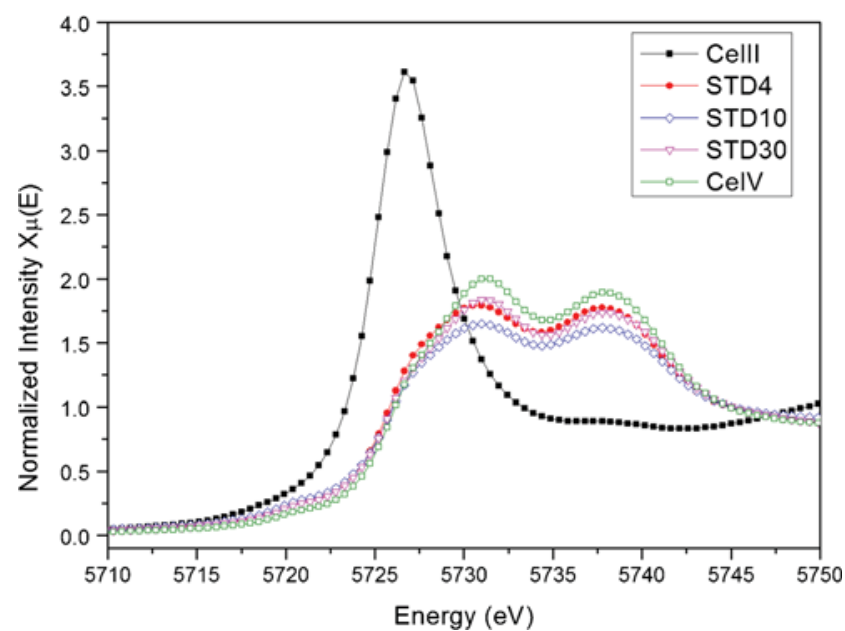

Fig. 3 High-energy resolution Ce-L3 XAS spectra (normalized signal versus energy) of the three nanoceria samples (STD-4, STD-10, STD$30)$ and two reference materials $\left(\mathrm{Ce}\left(\mathrm{NO}_{3}\right)_{3}\right.$ and $\left.\mathrm{CeO}_{2}\right)$ of the two oxidation state of cerium (Ce(III) and $\mathrm{Ce}(\mathrm{IV}))$, respectively
The UV-vis experiments were performed in three media: $\mathrm{HCl}$, ascorbic acid and hydrogen peroxide. They were selected due to its relationship with the applications of nanoceria as a catalyst [31], a free radical scavenger [26] and an oxidant in chemical reaction [32].

The UV-vis spectra in $0.1 \mathrm{M} \mathrm{HCl}$ showed as well significant differences between samples according to their different size [33]. STD-4 and STD-10 of similar size and similar Ce (III)/Ce(IV) ratio have similar UV-vis spectra, with two peaks at $264 \mathrm{~nm}$ and at $340 \mathrm{~nm}$ as correspond to small nanoparticles that have a relevant concentration of $\mathrm{Ce}$ (III) on the nanoparticles surface [31], while the spectrum of STD-30 showed a shoulder at $340 \mathrm{~nm}$ (Fig. 4), shifted to bigger values of wavelength as correspond to larger nanoparticles. This information is in agreement what it was previously described by XAS.

Ascorbic acid is commonly used as an antioxidant agent in food preparations and a common component of many physiological media that can interact with the $\mathrm{Ce}(\mathrm{IV})$ on the nanoceria surface, reducing it to Ce(III) [34]. Recently nanoceria has been proposed as drug delivery vehicle due to its ability to react with ascorbic acid and further dissolution of the reduced nanoparticle [33]:

$2 \mathrm{CeO}_{2}+3 \mathrm{C}_{6} \mathrm{H}_{8} \mathrm{O}_{6} \rightarrow \mathrm{Ce}_{2} \mathrm{O}_{3}+3 \mathrm{C}_{6} \mathrm{H}_{6} \mathrm{O}_{6}+\mathrm{H}_{2} \mathrm{O}$

Different concentrations of ascorbic acid were tested (2, 4, 6 and $8 \mathrm{mM}$ ) in $0.1 \mathrm{M} \mathrm{HCl}$. The spectra for an $8 \mathrm{mM}$ ascorbic acid at different times for the three samples are shown in Fig. 5. According to their spectra, the three studied samples reacted differently upon addition of ascorbic acid. Sample STD-4 turned orange when the ascorbic acid was added, although this colour faded away with time and became transparent after 45 min. Similarly, STD-10 turned into orange and maintained this colour longer after addition, not disappearing until $48 \mathrm{~h}$

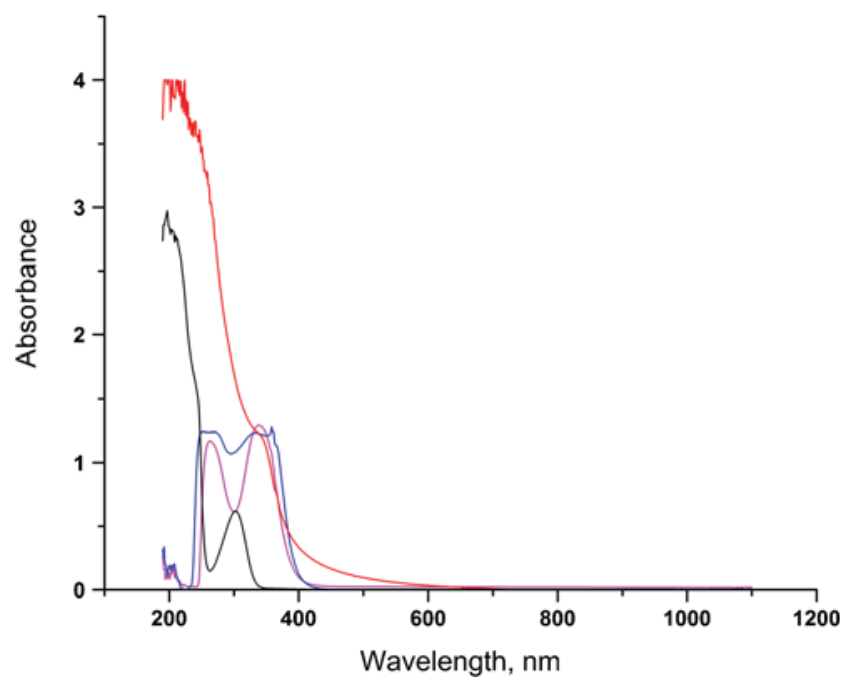

Fig. 4 UV-vis spectra in $0.1 \mathrm{M} \mathrm{HCl}$ for STD-4, $400 \mathrm{mg} / \mathrm{L}$ (in magenta); STD-10, $1400 \mathrm{mg} / \mathrm{L}$ (in blue); STD-30, $800 \mathrm{mg} / \mathrm{L}$ (in red); and $0.1 \mathrm{M}$ $\mathrm{HCl}$ blank solution (in black) 

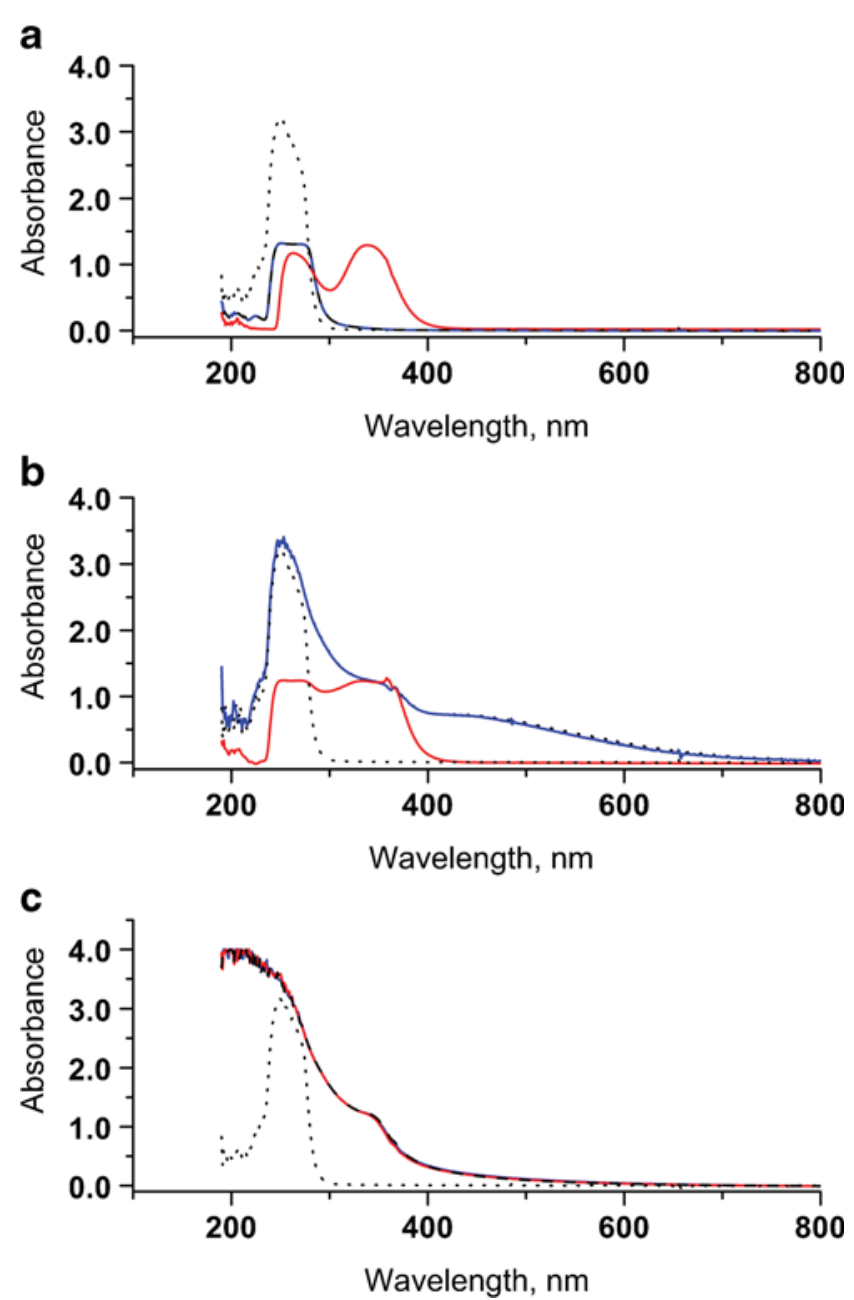

Fig. 5 Influence of ascorbic acid in the UV-vis spectra of the samples: A STD-4, $400 \mathrm{mg} / \mathrm{L}$; B STD-10, $1410 \mathrm{mg} / \mathrm{L}$; C STD-30, $816 \mathrm{mg} / \mathrm{L}$. Ascorbic acid $(8 \mathrm{mM})$, dotted line, nanoparticles in ascorbic acid- $0.1 \mathrm{M}$ $\mathrm{HCl}$ at $t=0$ in red, after $20 \mathrm{~min}$ of contact in blue

later. In contrast, STD-30 did not change its colour and the UVvis spectrum remains the same upon addition of the acid.

Sharpe et al. [34] consider that the de-hydroascorbic acid resulting of the oxidation of ascorbic acid is bound to the surface of the reduced CeNP. The resulting complex is responsible for a charge transfer effect that causes the colour change to brown. Their observation was supported by the changes in the UV-vis spectra of the CeNP before and after addition of the oxidant. The formation of complexes between carboxylic acids and the surface of a CeNP has been reported and studied by Lu et al. [35]. They concluded that the binding takes place by bidentate chelating and by bridging mode on the surface of the reduced NP.

The observed differences on the reaction rates with the ascorbic acid are due to the different size. The smaller nanoparticles (STD 4 and STD 10) exhibit a higher surface/volume ratio, which results in a faster reaction with the reducing agent. That is why STD 30 did not show any change when exposed to ascorbic acid. The differences of the reaction rate between STD 4 and STD 10 are likely due to the structural difference observed by Raman spectroscopy that makes STD 4 more reactive than STD 10.

Upon addition of $\mathrm{H}_{2} \mathrm{O}_{2}$, samples STD-4 and STD-10 were observed to turn orange indicating oxidation of the present $\mathrm{Ce}(\mathrm{III})$ to $\mathrm{Ce}(\mathrm{IV})$ and the formation of brown peroxyl complexes on the surface of the oxidized nanoparticle (32). In contrast, sample STD-30 did not show any change in colour during $48 \mathrm{~h}$ of observation. UV-vis spectra of the nanoparticles in the $\mathrm{HCl}-\mathrm{H}_{2} \mathrm{O}_{2}$ media were compared with those in $\mathrm{HCl}$ to explain this behaviour (Fig. 6). Some differences were noticed in the STD-4 spectra that were not observed in STD-10 and STD-30. For STD-4, the peak intensity at $337 \mathrm{~nm}$ corresponding to $\mathrm{Ce}(\mathrm{IV})$ decreased immediately after exposition to $\mathrm{H}_{2} \mathrm{O}_{2}$. This was interpreted as the reduction of $\mathrm{Ce}(\mathrm{IV})$ to Ce(III). For STD-10, the 337-nm peak shifted to $357 \mathrm{~nm}$ and with higher intensity upon $\mathrm{H}_{2} \mathrm{O}_{2}$ addition, while the peak at $261 \mathrm{~nm}$ decreased, indicating oxidation of Ce(III) to $\mathrm{Ce}$ (IV) in the nanoparticles. [36]. For STD-30, the spectrum in $\mathrm{HCl}$ did not virtually change upon addition of $\mathrm{H}_{2} \mathrm{O}_{2}$, suggesting
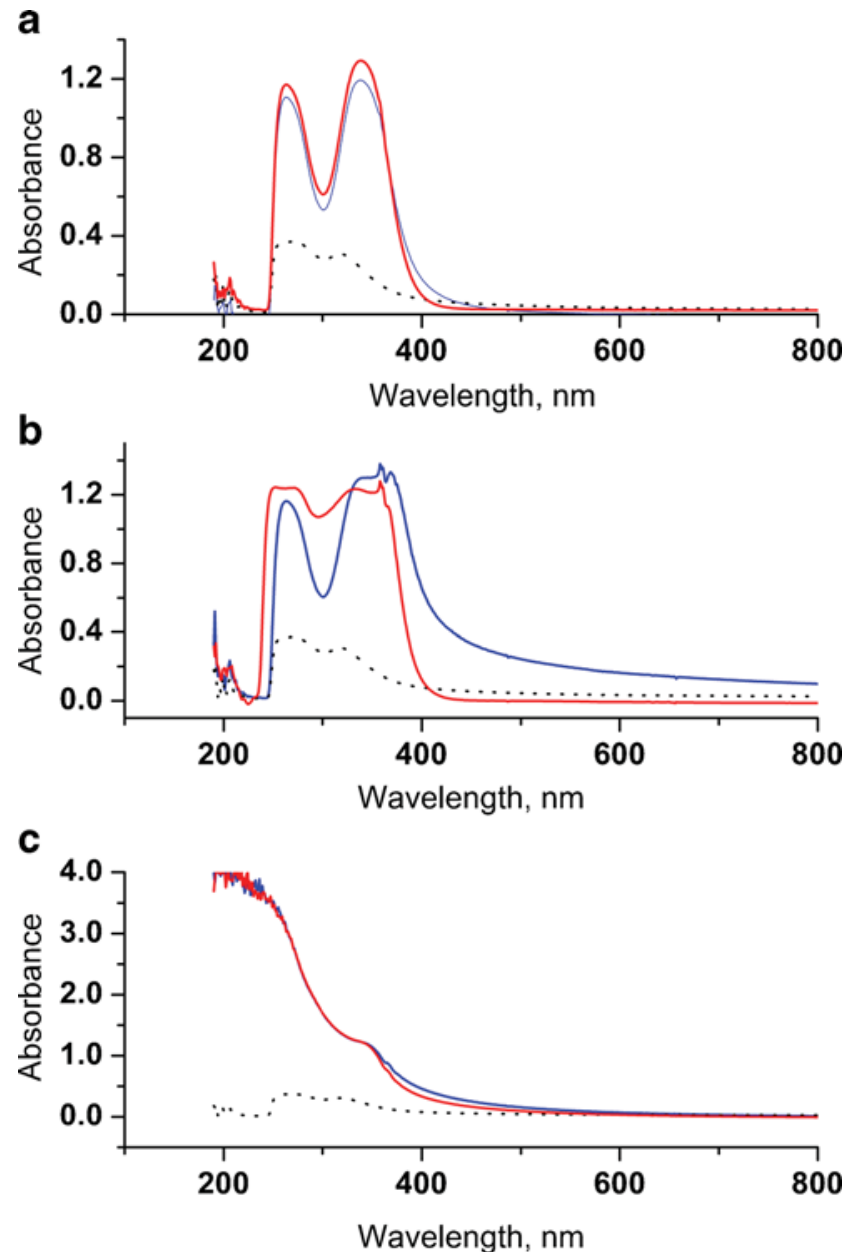

Fig. 6 Influence of hydrogen peroxide in the UV-vis spectra of the samples: A STD-4, 400 mg/L; B STD-10, $1410 \mathrm{mg} / \mathrm{L}$; C STD-30, $816 \mathrm{mg} / \mathrm{L}$. Blank $\left(10 \mathrm{mM} \mathrm{H}_{2} \mathrm{O}_{2}+0.1 \mathrm{M} \mathrm{HCl}\right)$ dotted line, nanoparticles at $t=0$ in red and at $t=20 \mathrm{~min}$ in blue 
that no significant reaction occurred with these nanoparticles. A similar lack of reaction was described elsewhere for $25 \mathrm{~nm}$ diameter nanoparticles [37]. The different behaviour observed between samples may be interpreted in the light of the observed differences in size and structure; bigger nanoparticles did not react with hydrogen peroxide while those with high degree of surficial defects result reduced by hydrogen peroxide in acidic medium.

PIC was used follow the evolution of nanoceria in suspensions under different chemical conditions and estimate their nanoparticle size, if possible. $\mathrm{Ce}(\mathrm{IV}) \mathrm{can}$ be reduced in aqueous media to $\mathrm{Ce}$ (III) following a process that is $\mathrm{pH}-$ and $\mathrm{O}_{2}$ concentration-dependent[38]. Since the cerium redox species mostly composing nanoceria is Ce(IV), we decided to use PIC to study the behaviour of the nanoparticles in suspension upon their reduction to $\mathrm{Ce}$ (III) in an oxygen-free solution by bubbling nitrogen through the solution for $10 \mathrm{~min}$. Then, a nitrogen blanket was kept on the solution surface to avoid reequilibration with the atmosphere.

Given the small dimensions of the nanoparticles, an evaluation of the size limit of detection was first required. This limit is determined by the noise produced by the instrument and the working electrode [39]. Here, blank experiments were run to measure the noise and the limit of detection was calculated as three times this noise. Two different microelectrode disc materials, Pt and carbon, were also investigated.

The minimum detectable nanoparticle diameter caused the smallest spike that could be observed. Its charge was estimated considering the spike as a rectangle triangle, the height three times the noise of the baseline and its base, the width of a regular spike, that is $10 \mathrm{~ms}$. The radii of the nanoparticle was then calculated using Eq. (1), if direct reduction of the nanoparticle is observed or using Eq. (2) if catalytic amplification is involved

$$
\begin{aligned}
& Q=4 F \pi \rho r^{3}{ }_{\mathrm{np}} / 3 A r=I \Delta t / 2 \\
& Q=4 \pi n F D r_{\mathrm{np}} \cdot \Delta t\left[\mathrm{H}_{2} \mathrm{O}_{2}\right]=I \Delta t / 2
\end{aligned}
$$

Where $Q$ is the charge of the collision event, $F$ the Faraday constant, $\rho$ the density of $\mathrm{CeO}_{2}, r_{\mathrm{np}}$ the nanoparticle radius and $A r$ the relative atomic mass.

The derived results for the smallest detectable spikes are shown in Table 2.

According to the defined limits of detection, the procedure does not allow us to detect the nanoparticles present in the samples under study. It has to be mentioned the improvement of the limit of detection in $0.1 \mathrm{M} \mathrm{HCl}+10 \mathrm{mM} \mathrm{H}_{2} \mathrm{O}_{2}$ media by increasing the magnitude of the detected signal using catalytic amplification [40] but it is not low enough to detect STD-4 and STD-10. However, the data proved useful to evaluate the formation of agglomerations in the three proposed media.
The first set of experiments was performed in $1 \mathrm{M} \mathrm{HCl}$. Working potentials of $0,-0.2$ and $-0.5 \mathrm{~V}$ were used. Collision events were observed only with the working electrode set at -0.5 V. A glassy carbon electrode was chosen as working electrode because the noise was very similar for both of them (see Table 2).

A diluted suspension of STD-4 was prepared in $1 \mathrm{M} \mathrm{HCL}$. A concentration of $4 \times 10^{18} \mathrm{NP} / \mathrm{L}(990 \mathrm{mg} / \mathrm{L}, 6.6 \mu \mathrm{M})$ was suspended in the electrochemical cell. After $520 \mathrm{~s}$ of observation, 43 spikes were obtained (Fig. 7)

The same experiment (observation time $520 \mathrm{~s}$ and working potential $-0.2 \mathrm{~V})$ was performed for STD-10 (4. $10^{18} \mathrm{NP} / \mathrm{L}$, $1410 \mathrm{mg} / \mathrm{L}, 6.6 \mu \mathrm{M})$ and STD-30 $\left(2.10^{14} \mathrm{NP} / \mathrm{L}, 816 \mathrm{mg} / \mathrm{L}\right.$, $0.3 \mathrm{nM})$. The concentration of the suspensions was limited by their stability in the supporting electrolyte. No single collision event was observed in neither case, indicating that almost all nanoparticles in the two samples had radii below the limit of detection of $27 \mathrm{~nm}$ (see Table 2). Extending the observation time to $1000 \mathrm{~s}$ did not increase the number of spikes. After $4000 \mathrm{~s}$, ample STD-30 produced only 11 peaks, while STD-10 chronoamperograms remained peakless. The radius of the detected STD-30 nanoparticles was calculated to be between 30 and $50 \mathrm{~nm}$ values that are above the nanoparticle diameters observed with TEM (i.e. 2 to $65 \mathrm{~nm}$ ). The low number of counts is also due to the low concentration of the nanoparticles, which lies in the nanomolar level.

In terms of agglomeration, PIC shows that STD-30 and STD-10 are stable in $1 \mathrm{HCl}$ even at high concentration (816 and $1410 \mathrm{mg} / \mathrm{L}$, respectively) for more than $1 \mathrm{~h}$. This is a key information with direct applications in e.g. medical studies that involve the oral intake of this nanoparticles along with water or contaminated food and their evolution in the $\mathrm{HCl}$ medium of the stomach. In spite of the similar size, shape and nanoparticle concentration of STD-4 relative to STD-10, nanoparticles in the former seemed more prone to aggregation in $\mathrm{HCl}$ since 43 collisions events of nanoparticles of 20 $40 \mathrm{~nm}$ of radius were observed in a shorter period of time. This could be explained by the structural differences (i.e. porosity) observed by Raman spectroscopy making the STD-4 nanoparticles more reactive.

The second set of PIC experiments were conducted upon addition of $8 \mathrm{mM}$ ascorbic acid to a suspension of STD-10 (4. $\left.10^{18} \mathrm{NP} / \mathrm{L}, 1410 \mathrm{mg} / \mathrm{L}, 6,6 \mu \mathrm{M}\right)$ and STD-30 $\left(2.10^{14} \mathrm{NP} / \mathrm{L}\right.$,

Table 2 PIC limits of detection for radius determination in three different media. n.m. stands for not measured. Each value is the mean of three measurements, $\mathrm{x} \pm \mathrm{s}$

\begin{tabular}{llll}
\hline $\begin{array}{l}\text { Working } \\
\text { electrode }(\mathrm{nm})\end{array}$ & $\begin{array}{l}1 \mathrm{M} \mathrm{HCl} \\
(\mathrm{nm})\end{array}$ & $\begin{array}{l}8 \mathrm{mM} \mathrm{AA} \\
(\mathrm{nm})\end{array}$ & $\begin{array}{l}0.1 \mathrm{M} \mathrm{HCl} \\
+10 \mathrm{mM} \mathrm{H}_{2} \mathrm{O}_{2}\end{array}$ \\
\hline $\mathrm{Pt}$ & $28 \pm 4$ & n.m. & $2 \pm 1$ \\
$\mathrm{GC}$ & $27 \pm 3$ & $32 \pm 2$ & n.m. \\
\hline
\end{tabular}


a

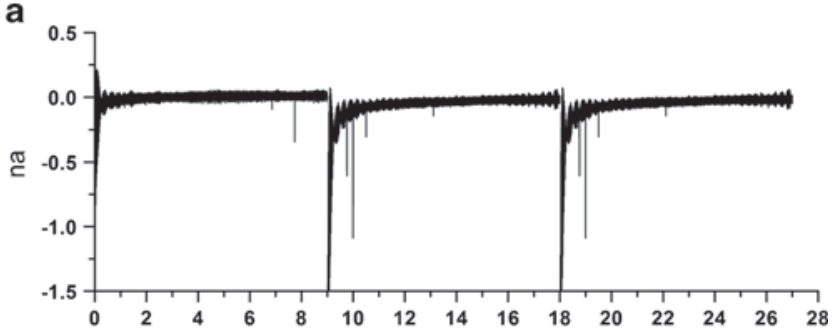

b

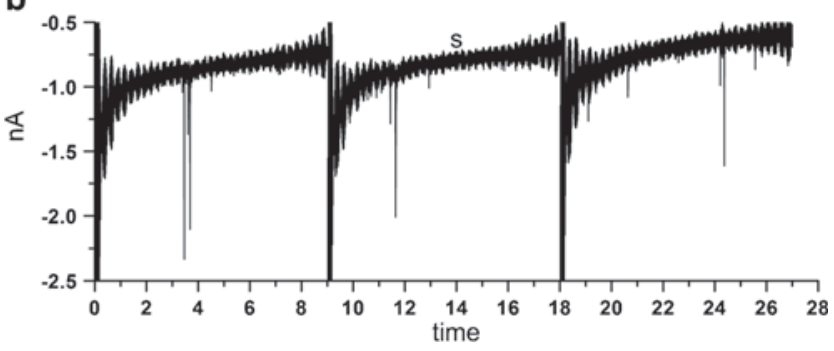

Fig. 7 Particle collisions events of STD-4 nanoparticles by direct reduction (a) and catalytic amplification (b). Three consecutive chronoamperograms for each type

$816 \mathrm{mg} / \mathrm{L}, 0.3 \mathrm{nM})$. STD-4 was not tested because it dissolved in this medium. No signal was observed by PIC, so we concluded that all the nanoparticles in samples STD-10 and STD30 are below $32 \mathrm{~nm}$ radius.

This is a valuable information with direct applications in for instance biomedical studies [33], where changes in the chemical behaviour of the ceria nanoparticles may be attributed to chemical alterations of the nanoparticle surface through interaction with other components, once the variation of the nanoparticles size (i.e. through aggregation or agglomeration) has been excluded.

A third and last set of experiments was performed upon addition of a catalyser that assists the reaction to amplify the electrochemical signal. $\mathrm{H}_{2} \mathrm{O}_{2}$ reduces $\mathrm{Ce}(\mathrm{IV})$ to $\mathrm{Ce}(\mathrm{III})$ at low $\mathrm{pH}$, and reoxidize it to $\mathrm{Ce}(\mathrm{IV})$ at high $\mathrm{pH}$ with increasing amount of $\mathrm{H}_{2} \mathrm{O}_{2}$ or with oxygen [33]. If the concentration of

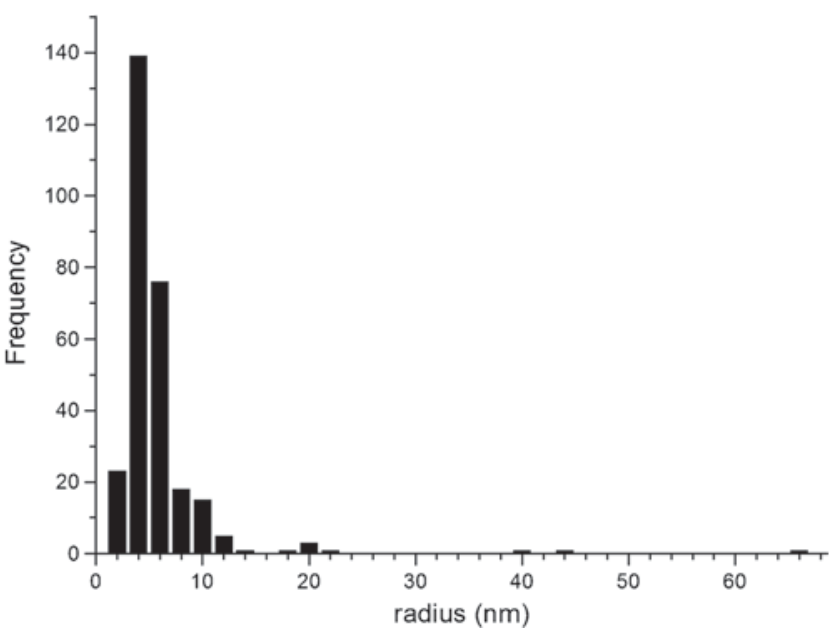

Fig. 8 Size distribution of the sample STD-4 using particle collision coulometry with catalytic amplification, $10 \mathrm{mM} \mathrm{H}_{2} \mathrm{O}_{2}$
$\mathrm{H}_{2} \mathrm{O}_{2}$ is below $100 \mathrm{mM}, \mathrm{Ce}(\mathrm{III})$ active sites are occupied by $\mathrm{H}_{2} \mathrm{O}_{2}$-like species forming stable complexes [41]. Sardesai et al. [42] detected such complexes by reducing them after collision of the ceria nanoparticles $(4 \mathrm{~g} / \mathrm{L})$ on a Pt microelectrode of $125 \mu \mathrm{m}$ diameter.

The ceria nanoparticles were treated with $10 \mathrm{mM} \mathrm{H}_{2} \mathrm{O}_{2}$ in $0.1 \mathrm{M} \mathrm{HCl}$ for $1.5 \mathrm{~h}$ (shorter time of treatment with $\mathrm{H}_{2} \mathrm{O}_{2}$ leads to unfruitful experiments) and then transferred to the electrochemical cell. The chronoamperograms were obtained at $-0.2 \mathrm{~V}$. In this case, the working electrode was a Pt microelectrode [35].

Collisions events were observed only for STD-4 (4 10 $10^{18}$ $\mathrm{NP} / \mathrm{L}, 990 \mathrm{mg} / \mathrm{L}, 6.6 \mu \mathrm{M}$ ) after $1.5 \mathrm{~h}$ of treatment with $\mathrm{H}_{2} \mathrm{O}_{2}$. In contrast, no spikes were detected for neither STD-10 (4 $\left.10^{18} \mathrm{NP} / \mathrm{L}, 990 \mathrm{mg} / \mathrm{L}, 6.6 \mu \mathrm{M}\right)$ nor STD-30 (2. $10^{14} \mathrm{NP} / \mathrm{L}$, $816 \mathrm{mg} / \mathrm{L}, 0.3 \mathrm{nM}$ ). For STD-4, 285 collision events were registered during a 1000-s observation time. The histogram of size distribution can be seen in Fig. 8 .

The size distribution derived from PIC showed that $90 \%$ of the STD-4 nanoparticles had radii smaller than $4 \mathrm{~nm}$, which are slightly larger than the values observed by TEM. As we could observe impacts of $2 \mathrm{~nm}$ radius nanoparticles too, we concluded that the addition of $\mathrm{H}_{2} \mathrm{O}_{2}$ may have caused mainly a dimers and trimers of the nanoparticles. To explain why similar nanoparticles like STD-10 did not produced the same response, one should bear in mind that STD-4 is a nanoparticle with different structure (i.e. more defects) than STD-10, as revealed by Raman spectroscopy, and consequently its capacity to form $\mathrm{OH}$ complexes responsible of the signal may be increased.

\section{Conclusions}

The combination of common techniques such as Raman and UV-vis spectroscopy with PIC constitutes a simple and easyto-operate methodology that can provide essential information about the structure and chemical properties of nanoceria, offering more data to select the adequate nanoparticle for each application.

A structured Raman band at $460 \mathrm{~cm}^{-1}$ and a UV-vis spectrum with two peaks at $260 \mathrm{~nm}$ and at $340 \mathrm{~nm}$ indicate higher content of $\mathrm{Ce}$ (III) in the nanoparticle, as it was confirmed by XAS. The presence of an additional band in the Raman spectrum at $595 \mathrm{~cm}^{-1}$ is a consequence of a higher number of defects in the crystal structure that makes this nanoparticle chemically more reactive than others of the same size and $\mathrm{Ce}(\mathrm{III})$ content.

PIC with catalytical amplification can detect nanoparticles down to $4 \mathrm{~nm}$ diameter, but only nanoparticles with more structural defects were detected. This factor plays an important role in the formation of the complex with the $\mathrm{OH}$ species that are responsible for the observed signal. 
Due to the high limits of detection for size determination using direct reduction of the nanoparticle, this technique was used to monitor aggregation/agglomeration of nanoparticles in media of interest for biomedical and environmental studies. The concentration of nanoparticles of the experiments is four times lower than the previously reported.

Acknowledgments This work has been supported by a Spanish competitive project CTQ 2015-68094-C2-1R.

The author Laura Sánchez-García acknowledges a postdoctoral research grant co-funded by the Fundación Agencia Aragonesa para la Investigación y Desarrollo de Aragón (ARAID) and Ramiro Córdova thanks the Instituto Universitario de Investigación en Ciencias Ambientales de Aragón (IUCA), Zaragoza University, for the scholarship "Apoyo a la Investigación" awarded for the 2014 academic year. We thank Diamond Light Source for access to beamline 18 (SP-11695) that contributed to the results presented here.

Thanks to Dr Mario Pérez Riera and Dr Francisco José Ruíz Blasco of the "Departamento de Matemáticas, Área de Análisis Matemático" who had helped us with Fourier transform smoothing of the chronoamperograms, without their help no collision events could have been ever seen in our lab. We also want to thank Dr Margarita Aznar and Dr Jesús Salafranca from the Analytical Chemistry Department of the University of Zaragoza for their help with the Raman spectra.

\section{Compliance with ethical standards}

Conflict of interest The authors declare that they have no conflict of interest.

\section{References}

1. Reed K, Cormack A, Kulkarni A, Mayton M, Sayle D, Klaessig F, et al. Exploring the properties and applications of nanoceria: is there still plenty of room at the bottom? Environ Sci Nano. 2014;1(5): 390-405.

2. Andreescu D, Bulbul G, Özel RE, Hayat A, Sardesai N, Andreescu S. Applications and implications of nanoceria reactivity: measurement tools and environmental impact. Environ Sci Nano. 2014;1(5):445-58.

3. Zlokovic BV. The blood-brain barrier in health and chronic neurodegenerative disorders. Neuron. 2008;57(2):178-201.

4. Roney C, Kulkarni P, Arora V, Antich P, Bonte F, Wu A, et al. Targeted nanoparticles for drug delivery through the blood-brain barrier for Alzheimer's disease. J Control Release. 2005;108(2-3): 193-214.

5. Bhaskar S, Tian F, Stoeger T, Kreyling W, de la Fuente JM, Grazú $\mathrm{V}$, et al. Multifunctional nanocarriers for diagnostics, drug delivery and targeted treatment across blood-brain barrier: perspectives on tracking and neuroimaging. Part Fibre Toxicol. 2010;7:3.

6. Lockman P, Koziara J, Mumper R, Allen D. Nanoparticle surface charges alter blood-brain barrier integrity and permeability. J Drug Target. 2004;12(9-10):635-41.

7. Xia T, Kovochich M, Brant J, Hotze M, Sempf J, Oberley T, et al. Comparison of the abilities of ambient and manufactured nanoparticles to induce cellular toxicity according to an oxidative stress paradigm. Nano Lett. 2006;6(8):1794-807.

8. Li N, Xia T, Nel AE. The role of oxidative stress in ambient particulate matter-induced lung diseases and its implications in the toxicity of engineered nanoparticles. Free Radic Biol Med. 2008;44(9):1689-99.

9. Pulido-Reyes G, Rodea-Palomares I, Das S, Sakthivel TS, Leganes F, Rosal R, et al. Untangling the biological effects of cerium oxide nanoparticles: the role of surface valence states. Sci Rep. 2015;5: 15613.

10. Gouadec G, Colomban P. Raman spectroscopy of nanomaterials: how spectra relate to disorder, particle size and mechanical properties. Progr Cryst Growth Char Mater 2007;1:1-56.

11. Xu R. Light scattering: a review of particle characterization applications. Particuology. 2015;18:11-21.

12. Singh S, Dosani T, Karakoti AS, Kumar A, Seal S, Self WT. A phosphate-dependent shift in redox state of cerium oxide nanoparticles and its effects on catalytic properties. Biomaterials. 2011;32(28):6745-53.

13. Peng Y, Chen X, Yi G, Gao Z. Mechanism of the oxidation of organic dyes in the presence of nanoceria. Chem Commun. 2011;47(10):2916-8.

14. Xiao X, Fan F-RF, Zhou J, Bard AJ. Current transients in single nanoparticle collision events. J Am Chem Soc. 2008;130(49): 16669-166677.

15. Cheng W, Compton RG. Electrochemical detection of nanoparticles by "nano-impact" methods. TrAC Trends Anal Chem. 2014;58:79-89.

16. Stuart EJE, Rees NV, Cullen JT, Compton RG. Direct electrochemical detection and sizing of silver nanoparticles in seawater media. Nanoscale. 2013;5(1):174-7.

17. Boika A, Bard AJ. Time of first arrival in electrochemical collision experiments as a measure of ultralow concentrations of analytes in solution. Anal Chem. 2015;87(8):4341-6.

18. Eloul S, Kätelhön E, Batchelor-McAuley C, Tschulik K, Compton RG. Diffusional impacts of nanoparticles on microdisc and microwire electrodes: the limit of detection and first passage statistics. J Electroanal Chem. 2015;755:136-42.

19. Toh HS, Compton RG. "Nano-impacts": an electrochemical technique for nanoparticle sizing in optically opaque solutions. Chem Open. 2015;4:261-3

20. Kätelhön E, Cheng W, Batchelor-McAuley C, Tschulik K, Compton RG. Nanoparticle-impact experiments are highly sensitive to the presence of adsorbed species on electrode surfaces. Chem Electro Chem. 2014;1(6):1057-62.

21. Batchelor-McAuley C, Ellison J, Tschulik K, Hurst PL, Boldt R, Compton RG. In situ nanoparticle sizing with zeptomole sensitivity. Analyst. 2015;140(15):5048-54.

22. Dent A, Cibin G, Ramos S, Parry S, Gianolio D, Smith A, et al. Performance of B18, the Core EXAFS bending magnet beamline at diamond. J Phys Conf Ser. 2013;430:1-7.

23. Ravel B, Newville M. ATHENA ARTEMIS HEPHAESTUS: data analysis for X-ray absorption spectroscopy using IFEFFIT. J Synchrotron Radiat. 2005;12:537-71.

24. Trogadas P, Parrondo J, Ramani V. CeO2 surface oxygen vacancy concentration governs in situ free radical scavenging efficacy in polymer electrolytes. ACS Appl Mater Interfaces. 2012;4(10): 5098-102.

25. Zhang F, Chan S, Spanier JE, Apak E, Jin Q, Robinson RD, et al. Cerium oxide nanoparticles : size-selective formation and structure analysis. Appl Phys Lett 2002;80(1):127-129.

26. Spanier J, Robinson R, Zhang F, Chan S-W, Herman I. Sizedependent properties of $\mathrm{CeO}_{2}-\mathrm{y}$ nanoparticles as studied by Raman scattering. Phys Rev B. 2001;64(24):245407.

27. Buckeridge J, Scanlon DO, Walsh A, Catlow CRA, Sokol AA. Dynamical response and instability in ceria under lattice expansion. Phys Rev B - Condens Matter Mater Phys. 2013;87(21):1-10.

28. Weber W, Hass K, McBride J. Raman study of $\mathrm{CeO}_{2}$. Second-order scattering, lattice dynamics and particle size effects. Phys Rev B Condens Matter 1993;48(1):178-85. 
29. Wu Z, Li M, Howe J, Meyer HM, Overbury SH. Probing defect sites on $\mathrm{CeO}_{2}$ nanocrystals with well-defined surface planes by raman spectroscopy and $\mathrm{O}_{2}$ adsorption. Langmuir. 2010;26(21): 16595-606.

30. Lee Y, He G, Akey AJ, Si R, Flytzani-stephanopoulos M, Herman IP. Raman analysis of mode softening in nanoparticle $\mathrm{CeO}_{2-\delta}$ and $\mathrm{Au}-\mathrm{CeO}_{2-\delta}$ during $\mathrm{CO}$ oxidation. J Am Chem Soc 2011;133(33)12952-12955.

31. Heckert EG, Karakoti AS, Seal S, Self WT. The role of cerium redox state in the SOD mimetic activity of nanoceria. Biomaterials. 2008;29(18):2705-9.

32. Ornatska M, Sharpe E, Andreescu D, Andreescu S. Paper bioassay based on ceria nanoparticles as colorimetric probes. Anal Chem. 2011;83(11):4273-80.

33. Muhammad F, Wang A, Qi W, Zhang S, Zhu G. Intracellular antioxidants dissolve man-made antioxidant nanoparticles: using redox vulnerability of nanoceria to develop a responsive drug delivery system. ACS Appl Mater Interfaces. 2014;6(21):19424-33.

34. Sharpe E, Frasco T, Andreescu D, Andreescu S. Portable ceria nanoparticle-based assay for rapid detection of food antioxidants (NanoCerac). Analyst. 2013;138(1):249-62.

35. Lu Z, Karakoti A, Velarde L, Wang W, Yang P, Thevuthasan S, et al. Dissociative binding of carboxylic acid ligand on nanoceria surface in aqueous solution: a joint in situ spectroscopic characterization and first-principles study. J Phys Chem C. 2013;117(46):24329-38.
36. Sayle TXT, Molinari M, Das S, Bhatta UM, Möbus G, Parker SC, et al. Environment-mediated structure, surface redox activity and reactivity of ceria nanoparticles. Nanoscale [Internet]. 2013;5(13): 6063-73.

37. Cafun J, Kvashnina KO, Casals E, Puntes VF, Glatzel P. Absence of $\mathrm{Ce}^{3+}$ sites in chemically active colloidal ceria nanoparticles. 2013;7(12):10726-32.

38. Hayes S a., Yu P, O'Keefe TJ, O'Keefe MJ, Stoffer JO. The phase stability of cerium species in aqueous systems. J Electrochem Soc. 2002;149(12):C623.

39. Tschulik K, Batchelor-McAuley C, Toh H-S, Stuart EJE, Compton RG. Electrochemical studies of silver nanoparticles: a guide for experimentalists and a perspective. Phys Chem Chem Phys. 2014;16(2):616-23.

40. Stuart EJEE, Rees NV, Compton RG. Particle-impact voltammetry: the reduction of hydrogen peroxide at silver nanoparticles impacting a carbon electrode. Chem Phys Lett. 2012;531:94-7.

41. Chen F, Shen X, Wang Y, Zhang J. $\mathrm{CeO}_{2} / \mathrm{H}_{2} \mathrm{O}_{2}$ system catalytic oxidation mechanism study via a kinetics investigation to the degradation of acid orange 7. Appl Catal B Environ . 2012;121-122: 223-9.

42. Sardesai NP, Andreescu D, Andreescu S. Electroanalytical evaluation of antioxidant activity of cerium oxide nanoparticles by nanoparticle collisions at microelectrodes. J Am Chem Soc. 2013;135(45):16770-3. 